\title{
Fast Non-Empiric Propeller Source Noise Model with Mean Flow
}

\author{
Andrea Franco ${ }^{(凶)}$, Roland Ewert, Malav Mukesh Soni, Michael Mößner, and \\ Jan Werner Delfs \\ Institute of Aerodynamics and Flow Technology, Dept. of Technical Acoustics \\ German Aerospace Center (DLR), Lilienthalplatz 7, 38108 Braunschweig, Germany, \\ andrea.franco@dlr.de
}

\begin{abstract}
A fast and physical-principles-based method to compute the noise radiation for installed propellers' applications is introduced. The simulation of the sound propagation includes newly defined perturbation equations, with propeller sound sources modelled as rotating singularities. The system of equations proposed is derived from the Linearised Euler Equations, after the application of a split-approach on the velocity perturbations. Two separate coupled systems of equations are obtained, the Acoustic Perturbation Equations, governing the acoustic velocity and pressure perturbations, and the Vortical Convection Equations, describing the convection of non-acoustic velocity perturbations. The moving sources are regularised with a Gaussian kernel over the computational mesh, allowing their description within an unstructured quadrature-free Discontinuous Galerkin experimental solver for Computational Aeroacoustics applications. The prediction capabilities of the method proposed have been successfully validated with a simple test case.
\end{abstract}

Keywords: CAA, Propeller Noise, APE+VCE

\section{Introduction}

The demand for novel sustainable aerial vehicles' concepts satisfying the EU Flightpath 2050 guidelines, opens new challenges for aircraft manufacturers concerning re-evaluation of the available propulsion technologies and aircraft re-design by means of unconventional propulsion integration configurations. Regardless of the configuration chosen, a requirement to fulfill for future aircraft concepts is a perceived reduction of $65 \%$ of noise emissions by 2050. Recent advances in aircraft propulsion configurations include distributed electric propulsion systems, and buried or semi-buried engine integrations on the airframe [1]. The noise abatement milestones are guiding research of propeller aeroacoustics in providing manufacturers with new design methods and tools capable of accurately and reliably estimating propeller noise emissions for any arbitrary arrangement. This work presents a fast and accurate method for computations of highly integrated propellers, developed from previous models of Gutin [2] and Lowson [3], who replaced the propeller blades by rotating source singularities. 
The method introduced is implemented in a Computational Aeroacoustics (CAA) framework to address highly non-uniform mean flows, as well as installation noise predictions. Source singularities avoid the resolution of the propeller geometry, increasing the method flexibility. A similar approach was considered by Dierke et.al. [9] in the finite difference block-structured CAA solver PIANO of DLR. In this work, the method is implemented within a quadrature-free Discontinuous Galerkin (DG) scheme, solving the governing equations on unstructured grids, thus, reducing the effort to generate computational meshes for highly complex geometries.

Alongside the proposed propeller model, a suitable robust system of equations for acoustic computations is required, which is capable of appropriately representing acoustic and vortical eigenmodes. Linearised Euler Equations (LEE) include the aforementioned eigenmodes and have been extensively used in the past for CAA applications; however, they would not be free from instabilities, such as the unbounded Kelvin-Helmholtz type. Motivated by the work of Goldstein [4], and further developments by Atassi et.al. [5] [6], a newly defined system of equations is presented, based on a velocity-split approach, separating acoustic and vortical modes as introduced in [7].

The propeller noise prediction method of this contribution is at the same time fast and physical-principles-based, and able to address both conventional and unconventional propulsion configurations, without requiring a priori empirical knowledge. Initial results of the implementation in the DG CAA experimental solver DISCO++ of DLR are reported. A rotating singularity is considered as a test case, to validate the concept against known analytical solutions. To address future installation noise predictions applications with arbitrary nonuniform inflow conditions, the rotating singularities model will be extended with line-distributed rotating source singularities of strengths determined from actuator disk RANS simulations.

\section{Propeller Source Noise Model Implementation}

The propeller source noise model is based on a rotating source singularity, representative either of loading or thickness noise contributions, or a combination of these two self-noise source components. This modelling choice allows to simulate a propeller without requiring moving surfaces or the exact blade geometry in the CAA simulations.

\subsection{Calculation of the Time Dependent Source Singularity Location}

Each propeller blade is replaced by a single source singularity, rotating around the propeller axis as time advances. The location of the singularities is calculated in a local cylindrical coordinate system, centered at the hub of the physical propeller to be modelled, with initial location $\mathbf{X}_{\mathbf{i n i t}}=\left(r_{\text {init }}, \phi_{\text {init }}, z_{\text {init }}\right)^{T}$ and updated circumferential coordinate $\phi_{\text {time }}$ computed from the source's Revolutions Per Second (RPS) prescribed in input, as seen in Fig. (1). 
Multiple propeller blades $N_{\text {blade }}$ are modelled through a circumferential offset $2 \pi / N_{\text {blade }}$ applied to $\phi_{\text {time }}$. The sources' values would be defined either by the integrated load vector $\mathbf{f}=\left(f_{r}, f_{\phi}, f_{z}\right)^{T}$ or the displacement $\dot{\theta}$ of the propeller on the air, and are included appropriately in the sources vector $\mathbf{S}=\left(S_{i}\right)^{T}$, where $i$ depends on the perturbation equation of interest.

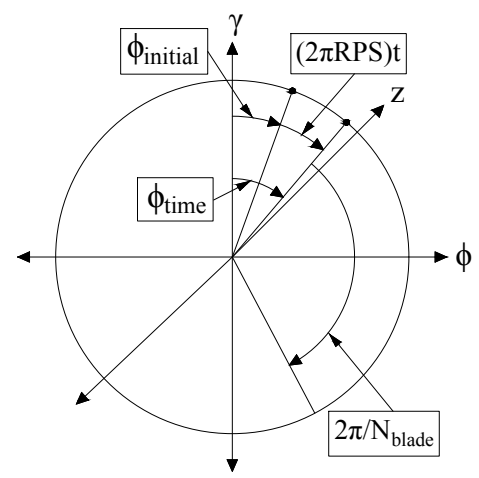

Fig. 1: Propeller cylindrical coordinate system

\subsection{Regularisation of Sources}

The definition of a singularity in space-discretised schemes, as the DG-method considered in this work, is a difficult task to consider, and is frequently the cause for numerical instabilities. Rather, the source must be approximated with a distributed value, which can be incorporated in the computational mesh. This is accomplished by regularising the values of the sources on the grid using a $3 \mathrm{D}$ isotropic Gaussian kernel $f(r, \phi, z)$ :

$$
f(r, \phi, z)=\left(\frac{\ln (2)}{\pi}\right)^{\frac{3}{2}} \frac{1}{\varepsilon} \exp \left(\frac{\ln \left(\frac{1}{2}\right) d^{2}}{\varepsilon^{2}}\right)
$$

where $d$ is the distance between the current position of the singularity in motion and a grid point, and $\varepsilon$ is the half-width at half-maximum of the Gaussian probability density function, representing the distance over which the Gaussian distribution drops from the maximum value to half its maximum. The integrated source values prescribed do not correspond to the required fluctuating source components to be included in perturbation equations. Instead they are described as the sum of a steady mean and a fluctuating component, where only the second component acts as a source in the perturbation equations.

For this reason, the steady mean value $\mathbf{S}_{\text {mean }}$ to be subtracted from the input source values is obtained by integrating over a circle the Gaussian regularised source. The resulting fluctuating component is obtained as $\mathbf{S}^{\prime}=\mathbf{S}-\mathbf{S}_{\text {mean }}$, and after being scaled for the perturbation equation of interest, it is assigned to the appropriate high order point determined by the DG discretisation. 


\section{System Of Perturbation Equations}

In this work, a newly defined system of equations combined with the propeller model is presented, which is suited to represent appropriately the interaction of acoustic and vortical eigenmodes, and is robust enough to address applications including complex geometries. The newly proposed system of equations is derived from the LEE, shown here in their primitive variables formulation:

$$
\begin{gathered}
\frac{\partial \rho^{\prime}}{\partial t}+\nabla \cdot\left(\rho_{0} \mathbf{u}^{\prime}+\rho^{\prime} \mathbf{u}_{\mathbf{0}}\right)=\dot{\theta}^{\prime} \\
\frac{\partial \mathbf{u}^{\prime}}{\partial t}+\left(\mathbf{u}_{\mathbf{0}} \cdot \nabla\right) \mathbf{u}^{\prime}+\left(\mathbf{u}^{\prime} \cdot \nabla\right) \mathbf{u}_{\mathbf{0}}+\frac{\nabla p^{\prime}}{\rho_{0}}-\rho^{\prime} \frac{\nabla p_{0}}{\rho_{0}^{2}}=\frac{\mathbf{f}^{\prime}}{\rho_{0}} \\
\frac{\partial p^{\prime}}{\partial t}+\mathbf{u}_{\mathbf{0}} \cdot \nabla p^{\prime}+\mathbf{u}^{\prime} \cdot \nabla p_{0}+\gamma p_{0} \nabla \cdot \mathbf{u}^{\prime}+\gamma p^{\prime} \nabla \cdot \mathbf{u}_{\mathbf{0}}=\frac{c_{0}^{2}}{\gamma} \dot{\theta}^{\prime}
\end{gathered}
$$

where $\rho, u, p, t$, and $\gamma$ are respectively the density, velocity, pressure, time, and specific heat ratio of air, with subscript 0 indicating mean flow quantities and primed symbols indicating perturbation quantities. Assuming isentropic flow conditions described by the linearised expression $p^{\prime}=\rho^{\prime} c_{0}^{2}$, with $c_{0}$ as the speed of sound computed from the mean flow, Eq. (2) can be rewritten:

$$
\frac{\partial p^{\prime}}{\partial t}+c_{0}^{2} \nabla \cdot\left(\mathbf{u}_{\mathbf{0}} \frac{p^{\prime}}{c_{0}^{2}}+\rho_{0} \mathbf{u}^{\prime}\right)=c_{0}^{2} \dot{\theta}^{\prime}
$$

As a consequence of introducing Eq. (5), Eq. (4) can be neglected and, including the isentropic relation written as $\rho^{\prime}=p^{\prime} / c_{0}^{2}$, the isentropic formulation of the LEE (LEE-s) reads:

$$
\begin{gathered}
\frac{\partial p^{\prime}}{\partial t}+c_{0}^{2} \nabla \cdot\left(\mathbf{u}_{\mathbf{0}} \frac{p^{\prime}}{c_{0}^{2}}+\rho_{0} \mathbf{u}^{\prime}\right)=c_{0}^{2} \dot{\theta}^{\prime} \\
\frac{\partial \mathbf{u}^{\prime}}{\partial t}+\left(\mathbf{u}_{\mathbf{0}} \cdot \nabla\right) \mathbf{u}^{\prime}+\left(\mathbf{u}^{\prime} \cdot \nabla\right) \mathbf{u}_{\mathbf{0}}+\nabla\left(\frac{p^{\prime}}{\rho_{0}}\right)=\frac{\mathbf{f}^{\prime}}{\rho_{0}} \\
\rho^{\prime}=\frac{p^{\prime}}{c_{0}^{2}}
\end{gathered}
$$

The proposed newly defined system of equations is obtained from Eq. (6), Eq. (7), and Eq. (8), by rewriting Eq. (7) using the mathematical identity:

$$
\left(\mathbf{u}_{0} \cdot \nabla\right) \mathbf{u}^{\prime}+\left(\mathbf{u}^{\prime} \cdot \nabla\right) \mathbf{u}_{0}=\nabla\left(\mathbf{u}^{\prime} \cdot \mathbf{u}_{0}\right)+\omega^{\prime} \times \mathbf{u}_{0}+\omega_{0} \times \mathbf{u}^{\prime}
$$

with $\boldsymbol{\omega}^{\prime}=\nabla \times \mathbf{u}^{\prime}$ and $\boldsymbol{\omega}_{\mathbf{0}}=\nabla \times \mathbf{u}_{\mathbf{0}}$. The perturbation velocities $\mathbf{u}^{\prime}$ of Eq. (7) are then split as $\mathbf{u}^{\prime}=\mathbf{u}^{\mathbf{a}}+\mathbf{u}^{\mathbf{r}}$, where $\mathbf{u}^{\mathbf{a}}=\nabla \varphi$ is the potential (acoustic) component and $\mathbf{u}^{\mathbf{r}}$ is the non-acoustic component including the information of the initial solenoidal field of the LEE as well. In the resulting split momentum equation for $\mathbf{u}^{\mathbf{a}}$, the term $\boldsymbol{\omega}^{\mathbf{a}} \times \mathbf{u}_{\mathbf{0}}$ is identically null, since $\boldsymbol{\omega}_{\mathbf{a}}=\nabla \times \mathbf{u}_{\mathbf{a}}=\mathbf{0}$. 
Re-arranging the terms among the split momentum equations, the system of equations reads:

$$
\begin{gathered}
\frac{\partial p^{\prime}}{\partial t}+c_{0}^{2} \nabla \cdot\left(\mathbf{u}_{\mathbf{0}} \frac{p^{\prime}}{c_{0}^{2}}+\rho_{0} \mathbf{u}^{\mathbf{a}}\right)=-c_{0}^{2} \nabla \cdot\left(\rho_{0} \mathbf{u}^{\mathbf{r}}\right)+c_{0}^{2} \dot{\theta}^{\prime} \\
\frac{\partial \mathbf{u}^{\mathbf{a}}}{\partial t}+\nabla\left(\mathbf{u}^{\mathbf{a}} \cdot \mathbf{u}_{\mathbf{0}}\right)+\nabla\left(\frac{p^{\prime}}{\rho_{0}}\right)=-\mathbf{X} \\
\frac{\partial \mathbf{u}^{\mathbf{r}}}{\partial t}+\left(\mathbf{u}_{\mathbf{0}} \cdot \nabla\right) \mathbf{u}^{\mathbf{r}}+\left(\mathbf{u}^{\mathbf{r}} \cdot \nabla\right) \mathbf{u}_{\mathbf{0}}=-\boldsymbol{\omega}_{\mathbf{0}} \times \mathbf{u}^{\mathbf{a}}+\mathbf{X}+\frac{\mathbf{f}^{\prime}}{\rho_{0}}
\end{gathered}
$$

Equation (10) and Eq. (11), without the source term $-c_{0}^{2} \nabla \cdot\left(\rho_{0} \mathbf{u}^{\mathbf{r}}\right)$, are the APE-4 [8] as being used in CAA to predict the propagation of acoustic modes over a non-uniform medium. Equation (12) is an additional transport equation, hereafter referred to as Vortical Convection Equation (VCE), representing the convection of non-acoustic velocity perturbations on a background mean flow. The system of equations composed of Eq. (10), Eq. (11), and Eq. (12) is named $\mathrm{APE}+\mathrm{VCE}$, and is equivalent to the LEE-s, as it can be verified by adding Eq. (11) with Eq. (12). Furthermore, the velocity splitting is not required to provide exactly distinct potential and solenoidal components of the velocity, as described in [7]. To formulate a mathematically equivalent system of equations to the LEE$\mathrm{s}$, the vector field $\mathbf{X}$ was introduced, without any assumption being made on its nature with respect to the eigenmodes of the equation. $\mathbf{X}$ can be freely defined to suit a specific velocity-split definition, hence, the splitting is general and can be flexibly used for installation noise prediction purposes, in which vortical and acoustic eigensolutions are non-distinct. In the remainder of this work, it was decided to set $\mathbf{X}=0$, in order to avoid the introduction of additional sources in Eq. (11), thus, verifying the possible application of the proposed equations to simplified test cases. The original fluctuating source terms of the LEE can be assigned to the RHS of Eq. (10) for externally prescribed mass sources, and to the RHS of Eq. (12) for externally prescribed fluctuating forces.

\section{Validation}

The proposed method, combining the propeller model with the APE+VCE, has been validated, and the results are shown in the form of polar directivities and a narrow band spectrum of the fluctuating pressure signal. Tests have been conducted for both a rotating force and mass singularity, in a uniform flow aligned with the axis of rotation of the sources. A sensitivity study for the appropriate choice of $\varepsilon$ was considered, and the results are presented as a comparison of polar directivities for different chosen frequency modes. Additional comparisons involve the solution of various perturbation equations, viz. LEE (Eq. (2), Eq. (3), Eq. (4)), LEE-s (Eq. (6), Eq. (7), Eq. (8)), APE+VCE (Eq. (10), Eq. (11), Eq. (12)), and $\operatorname{APE}$ (Eq. (10), Eq. (11) without $\left.-c_{0}^{2} \nabla \cdot\left(\rho_{0} \mathbf{u}^{\mathbf{r}}\right)\right)$. 


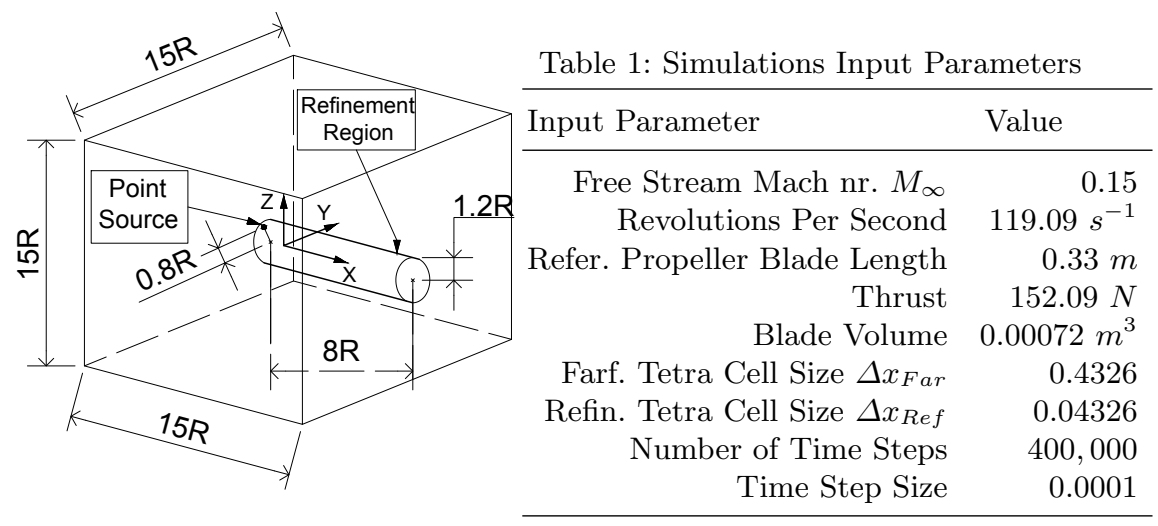

Fig. 2: Computational Domain

The computational domain is sketched in Fig. (2). Table (1) summarises the simulation input parameters, including the strengths of the source singularities. To resolve the source singularities regularised with Eq. (1), a cylindrical refinement region is generated, with a radial extension chosen to prevent a sharp transition of grid cells' size in the area of vortex propagation. Within the refinement region, each type of singularity is located at $0.8 \mathrm{R}$ in the radial direction to best represent loading and blade thickness effects for a propeller. A non-reflecting boundary condition, based on an upwind flux vector splitting approach, with prescribed null incoming eigenmodes, is applied on the domain outer surfaces. The computational time was approximately equivalent to four complete revolutions of the rotating singularity. $\Delta x_{F a r}$ was chosen to resolve the first ten harmonics. All the results are shown in terms of Sound Pressure Level (SPL), considering a ratio of $\varepsilon / \Delta x_{R e f}=2$, to appropriately resolve the regularised source on the computational grid. To better appreciate the energy content of the solution, each SPL result is described considering the 1st, 3rd, and 5th frequency mode of the fluctuating pressure signals, captured on the $\mathrm{z}$-x coordinate plane. Microphones are placed at a distance of $10 \mathrm{R}$ from the origin of the cylindrical coordinate system of the propeller. The results obtained from CAA simulations are compared with related results from known analytical equations for moving source singularities, formulated either in presence or absence of flow. Equation (13a) and Eq. (13b) describe respectively the pressure perturbation field for a given mass $\theta_{p}$ or force singularity $\mathbf{f}_{\mathrm{p}}$.

$$
\begin{array}{r}
p_{\theta_{p}}^{\prime}(\mathbf{x}, t)=\frac{1}{4 \pi}\left\{\frac{\frac{\partial \theta_{p}}{\partial \tau}+\mathbf{e}_{\mathbf{R}} \cdot \frac{\partial \mathbf{M}_{\mathbf{q}}}{\partial \tau}\left(1-M_{q R}\right)^{-1} \theta_{p}}{R_{i}\left(1-M_{q R}\right)^{2}}+\frac{a_{\infty}\left(M_{q R}-\mathbf{M}_{q}^{2}\right)}{R_{i}^{2}\left(1-M_{q R}\right)^{3}} \theta_{p}\right\} \\
p_{f_{p}}^{\prime}(\mathbf{x}, t)= \\
\frac{1}{4 \pi}\left\{\frac{\frac{\partial \mathbf{f}_{\mathbf{p}}}{\partial \tau} \cdot \mathbf{e}_{\mathbf{R}}+\left(\mathbf{f}_{\mathbf{p}} \cdot \mathbf{e}_{\mathbf{R}}\right) \frac{\partial \mathbf{M}_{\mathbf{q}}}{\partial \tau} \cdot \mathbf{e}_{\mathbf{R}}\left(1-M_{q R}\right)^{-1}}{a_{\infty} R_{i}\left(1-M_{q R}\right)^{2}}\right\}+ \\
+\frac{1}{4 \pi}\left\{\frac{-\mathbf{f}_{\mathbf{p}} \cdot \mathbf{M}_{\mathbf{q}}+\left(1-\mathbf{M}_{\mathbf{q}}{ }^{2}\right) \mathbf{f}_{\mathbf{p}} \cdot \mathbf{e}_{\mathbf{R}}\left(1-M_{q R}\right)^{-1}}{R_{i}^{2}\left(1-M_{q R}\right)^{2}}\right\}
\end{array}
$$


$\mathbf{M}_{\mathbf{q}}$ represents the Mach number of the source motion, $\mathbf{x}$ the observer position, $\mathbf{y}(\boldsymbol{\tau})$ the singularity position dependent on the retarded time $\tau, R_{i}=\left|\mathbf{R}_{\mathbf{i}}\right|=$ $|\mathbf{x}-\mathbf{y}(\tau)|$ the distance between source and observer, $\mathbf{e}_{\mathbf{R}}=\mathbf{R}_{\mathbf{i}} / R_{i}$ the unit distance vector between source and observer, $\mathbf{M}_{\mathbf{q R}}=\mathbf{M}_{\mathbf{q}} \cdot \mathbf{e}_{\mathbf{R}}$ the momentary Mach number component in the direction of the observer, and $a_{\infty}$ the speed of sound. If a uniform flow is considered, the distance $R_{i}$ will change as $R_{i}=$ $\left|\mathbf{R}_{\mathbf{i}}\right|=\left|\mathbf{x}-\mathbf{y}(\boldsymbol{\tau})-\mathbf{U}_{\infty}(\tau-t)\right|$, and the Mach number will change as well as $\mathbf{M}_{\mathbf{q}}=\mathbf{M}_{\mathbf{q}}+\mathbf{U}_{\infty}$, with $\mathbf{U}_{\infty}$ representing the free stream velocity vector.

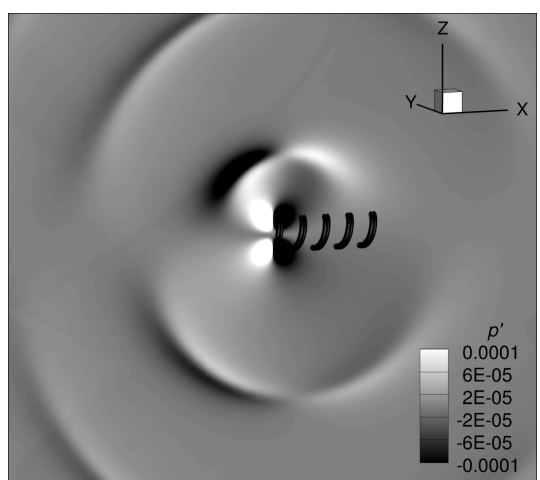

Fig. 3: Contour plot of the fluctuating pressure for simulated thrust singularity, with Q-Criterion superimposed

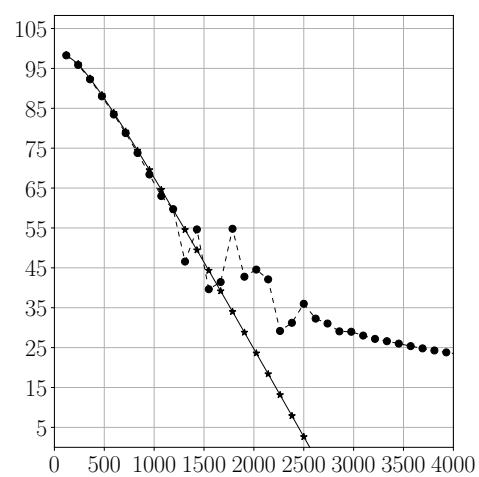

Fig. 4: Narrow band spectrum of $p^{\prime}$, simulated thrust, $M_{\infty}=0.15$. Horizontal axis: frequency $(\mathrm{Hz})$, Vertical axis: SPL (dB). The lines in the plot correspond to Analytical results (solid), $\mathrm{APE}+\mathrm{VCE}$ results (dash)

A qualitative inspection of the results of a simulated thrust force with the $\mathrm{APE}+\mathrm{VCE}$ system of equations exhibits the fundamental features to be expected from the simplified test case investigated. Figure (3) presents the pressure fluctuations contour, with Q-Criterion iso-surfaces superimposed, computed from $\mathbf{u}^{\mathbf{r}}$. The proposed system of equations describes qualitatively well the radiated sound pattern. Quadrupole-type pressure fluctuation patterns can be observed. A ring vortex pattern, generated due to the rotation of the force singularity, results from the Q-Criterion iso-surfaces. An important feature of propeller noise prediction-methods is the ability to capture the logarithmic decreasing trend of the harmonics, as highlighted in Fig. (4), where the narrow band spectrum of the fluctuating pressure signal at a location of $45^{\circ}$ is shown. The results are obtained simulating a thrust force with $M_{\infty}=0.15$. Each marker in the plot refers to a single harmonic. The SPL of the first 10 harmonics presents the expected rapid decreasing trend in $\mathrm{dB}$ values, as higher harmonics are investigated. 


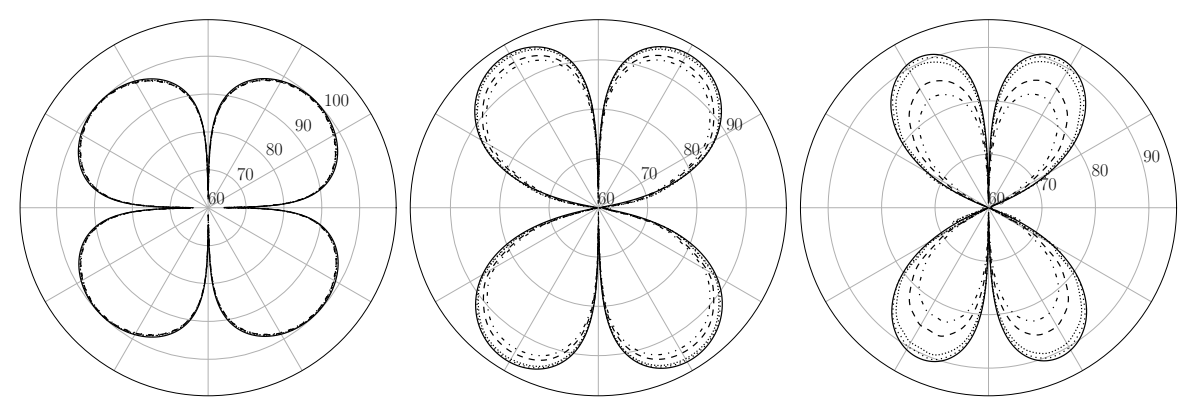

Fig. 5: SPL (dB) for different $\varepsilon / \Delta x_{R e f}$. Simulated thrust with $M_{\infty}=0$. From left to right are shown the 1st, 3rd and 5th frequency mode. The lines in each plot correspond to: Analytical results (solid), $\varepsilon / \Delta x_{R e f}=2$ (loose dot), $\varepsilon / \Delta x_{R e f}=4$ (dot), $\varepsilon / \Delta x_{R e f}=8$ (dash), and $\varepsilon / \Delta x_{R e f}=10$ (dashdot)

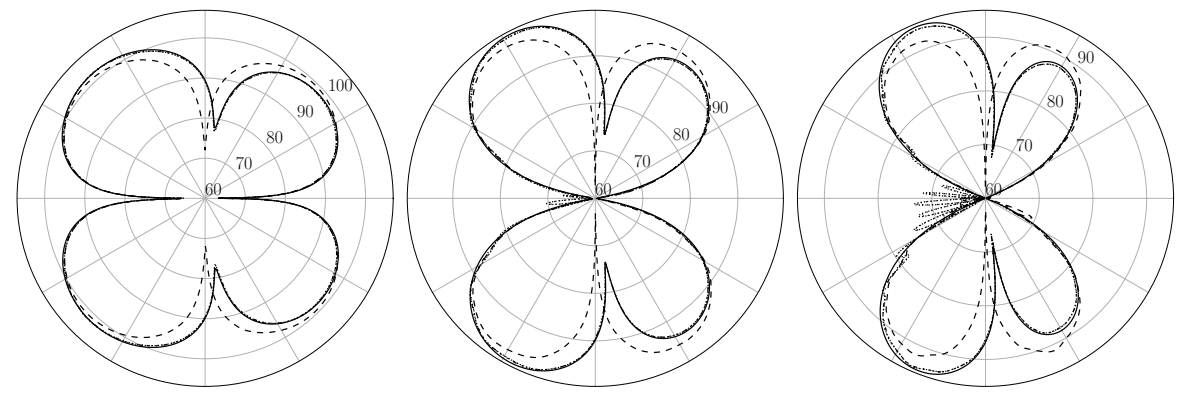

Fig. 6: SPL (dB) equations comparison. Results of a simulated thrust with $M_{\infty}=$ 0.15. From left to right are shown the 1st, 3rd and 5th frequency mode. The lines in each plot correspond to: Analytical results (solid), LEE (loose dot), LEE-s (dot), $\mathrm{APE}+\mathrm{VCE}$ (dashdot), and APE (dash)

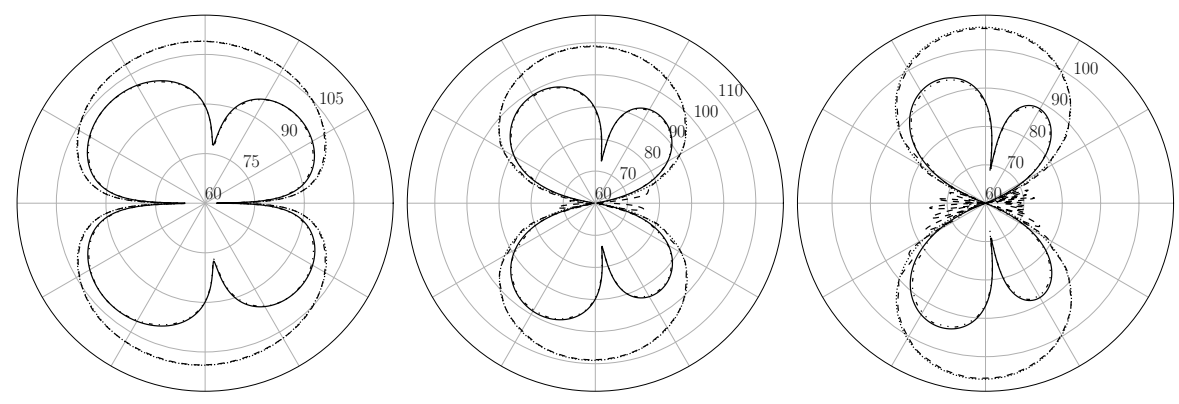

Fig. 7: SPL (dB) Thrust and mass singularities comparison, with $M_{\infty}=0.15$.From left to right are shown the 1st, 3rd and 5th frequency mode. The lines in each plot correspond to: Analytical Thrust results (solid), Analytical Mass results (dot), APE+VCE Mass results (dash), APE+VCE Thrust results (dashdotdot) 
For frequencies higher than $1100 \mathrm{~Hz}$, large SPL values are seen, which might be due to a limited mesh resolution or spurious numerical noise. In Fig. (5), the results of a sensitivity study for different ratios $\varepsilon / \Delta x_{R e f}$ are shown, with a single thrust force source in the APE $+\mathrm{VCE}$ equations and with $M_{\infty}=0$. For the 1st mode, the louder levels are observed at around $45^{\circ}$ in the first quadrant with symmetrical values in other quadrants. Higher modes show a shift of the maximum levels location towards $90^{\circ}$ and $270^{\circ}$. The energy content of the 1 st mode is seen not to depend on the choice of $\varepsilon / \Delta x_{R e f}$. For the 3rd mode, the SPL becomes sensitive towards $\varepsilon / \Delta x_{R e f}$ ratios, showing that larger values of $\varepsilon / \Delta x_{R e f}$ influence the higher harmonics. When analyzing the results of the 5 th mode, the differences obtained with different $\varepsilon / \Delta x_{R e f}$ are clearly visible: values up to $\varepsilon / \Delta x_{R e f}=4$ show similar levels, whereas, higher $\varepsilon / \Delta x_{R e f}$ produce a reduction in SPL. This indicates how a singularity, regularised over several computational grid points, would exhibit a modification of the radiated sound wave with a considerable reduction in its energy content. Microphones have been placed at a distance 10R to avoid the nearfield effects. Axial noise emission, as seen from the SPL of the 5th mode, indicates the presence of numerical noise otherwise not present from analytically predicted results. The equivalence of the APE+VCE with the LEE-s and LEE, as stated in Sect. (3), is verified with a simplified test case simulating a single thrust force with $M_{\infty}=0.15$. The results included in Fig.(6) in terms of SPL, show a comparison of these equations together with analytical and APE solutions. The results of all the equations, except for the case of APE, show a very good agreement of the SPLs for the 1st mode. The expected Doppler shift of the directivity pattern is not seen in the results of APE. Due to the filtering of entropical and vortical modes applied in their formal derivation, the APE would require an appropriate source definition to restore the correct prediction of the pressure signal [8]. In the APE+VCE formulation, this requirement is highlighted by the source term present in Eq. 10. The comparison of different equations for the 3rd and 5th frequency mode maintains overall a very good agreement, APE results aside. Some excess noise is detectable in the vicinity of the axis of rotation of the force singularity. A similar explanation as provided for the excess noise seen in Fig. (5) can be considered also in this case. Other than a thrust force, a mass source singularity was also simulated, to qualitatively verify the prediction capabilities of the method. Figure (7) shows the results in terms of SPL, obtained with $M_{\infty}=0.15$. The directivity patterns of all the frequency modes are represented as expected. As the mode number increases, excess noise is visible at locations close to the rotational axis of the singularity, as seen also in Fig. (5).

\section{Conclusions and Future Outlook}

A new propeller noise prediction method was developed in a CAA framework, including regularised force and mass sources singularities, as well as the newly proposed APE $+\mathrm{VCE}$ system of perturbation equations. 
The proposed method aims to reduce, at an acceptable level of accuracy, the computational cost of CAA simulations for propeller noise estimation, in order to investigate installation noise. Initial results of this approach show the expected trends and patterns of the predicted fluctuating pressure signal. SPLs of simulated thrust and mass singularities, in a uniform background medium, capture the essential noise prediction features. Some excess numerical noise is seen to be present near the rotational axis of the singularities. As a future development, numerical noise mitigation techniques will be included, and the model will be extended to line-distributed rotating source singularities.

\section{Acknowledgements}

We would like to acknowledge the funding by the Deutsche Forschungsgemeinschaft (DFG, German Research Foundation) under Germany's Excellence Strategy - EXC 2163/1 - Sustainable and Energy Efficient Aviation - Project-ID 390881007

\section{References}

1. Gohardani, A. S., Doulgeris, G., and Singh, R.: Challenges of future aircraft propulsion: A review of distributed propulsion technology and its potential application for the all electric commercial aircraft, Progress in Aerospace Sciences, Vol. 47, No.5, 2011, pp. 369, 391. doi:10.1016/j.paerosci.2010.09.001

2. Gutin, L.: On the Sound Field of a Rotating Propeller, NACA TM-1195, 1948.

3. Lowson, M. V., and Lighthill, M. J., "The sound field for singularities in motion," Proceedings of the Royal Society of London, Vol. 286, No. 1407, 3 Aug. 1965, pp. 559-572. doi:10.1098/rspa.1965.0164

4. Goldstein, M. E.: Unsteady vortical and entropic distortions of potential flows round arbitrary obstacles, Journal of Fluid Mechanics, Vol. 89, No. 3, 1978, pp. 433-468. doi:10.1017/S0022112078002682

5. Atassi, H. M., and Golubev, V. V.: Unsteady Disturbances in Swirling Turbomachinery Flows, Unsteady Aerodynamics and Aeroelasticity of Turbomachines, edited by T.H. Fransson, Springer, Dordrecht., 1998. doi:10.1007/978-94-011-5040-8_10

6. Logue, M., and Atassi, H.: Scattering of Acoustic Waves by a Rotor, 14th AIAA/CEAS Aeroacoustics Conference, AIAA, Vancouver, CA, 2008. doi:10.2514/ 6.2008-2989

7. Ewert, R., and Kreuzinger, J.: Hydrodynamic/acoustic splitting approach with flowacoustic feedback for universal subsonic noise computation, Preprint submitted to Journal of Computational Physics, 2020. URL:https://arxiv.org/abs/2009.07155

8. Ewert, R., and Schroeder, W.: Acoustic perturbation equations based on flow decomposition via source filtering, Journal of Computational Physics, Vol. 188, No. 2, 2003, pp. 365-398. doi:10.1016/S0021-9991(03)00168-2

9. Dierke, J., Akkermans, R. A., Delfs, J. W., and Ewert, R.: Installation Effects of a Propeller Mounted on a Wing with Coanda Flap. Part II: Numerical Investigation and Experimental Validation, 20th AIAA/CEAS Aeroacoustics Conference, Atlanta, GA, 2014. doi:10.2514/6.2014-3189 